\title{
SPECTRAL ANALYSIS OF THE LUMINOSITY DISTRIBUTION OF SEVEN GALAXIES IN THE VIRGO CLUSTER
}

Masanori Iye

Tokyo Astronomical Observatory, Mitaka 181 JAPAN

An objective method involving a Fourier analysis is applied to seven galaxies for measuring the strength of spiral and bar components.

A spectral analysis of the spiral patterns of galaxies is proposed by Iye et al.(1979). In this scheme, we define a Fourier transform $A(\alpha, m)$ of the intensity distribution $I(u=\ln r, \theta)$ of a galaxy by

$$
A(\alpha, m)=\frac{1}{2 \pi} \int_{0}^{2 \pi} \int_{-\infty}^{\infty} I(u, \theta) \exp \{-i(\alpha u+m \theta)\} d u d \theta,
$$

and the normalized power spectrum $P(\alpha, m)$ by

$$
P(\alpha, m)=|A(\alpha, m)|^{2} / \sum_{m=1}^{\infty} \int_{-\infty}^{\infty}|A(\alpha, m)|^{2} d \alpha \text {. }
$$

The application of this method to an asymmetric spiral galaxy NGC 4254 revealed that the spiral pattern of NGC 4254 is a superposition of fivearmed and three-armed spiral components on the main one-armed spiral (Iye et al. 1982).

In the present paper, the same method is applied to seven galaxies NGC 4303 (SCI), NGC 4321 (SCI), NGC 4340 (SBa), NGC 4472 (E4), NGC 4501 $\left(\mathrm{Sb}^{+} \mathrm{I}\right), \mathrm{NGC} 4535(\mathrm{~S}(\mathrm{~B}) \mathrm{CI}:)$, and NGC 4579 (Sbn). Figures 1, 2, and 3 show the $\mathrm{V}$-band image, the power spectrum $P(\alpha, m)$, and the pattern of the primary spectral component, respectively, of two spiral galaxies NGC 4303 and NGC 4535. The primary spiral component of NGC 4535 is very strong and this singly reproduces the overall spiral feature of NGC 4535 (Figure 3b). On the other hand, the spiral feature of NGC 4303 is composed of a few spiral components of which the strongest is shown in Figure $3 a$.

\section{REFERENCES}

Iye, M., Hamabe,. M., Watanabe, M., and Okamura, s.: 1979, in Photometry, Kinematics and Dynamics of Galaxies, ed. D. S. Evans, University of Texas, pp.407-410.

Iye, M., Okamura, S., Hamabe, M., and Watanabe, M.: 1982, Astrophys. J. 256, pp.103-111. 
(a)

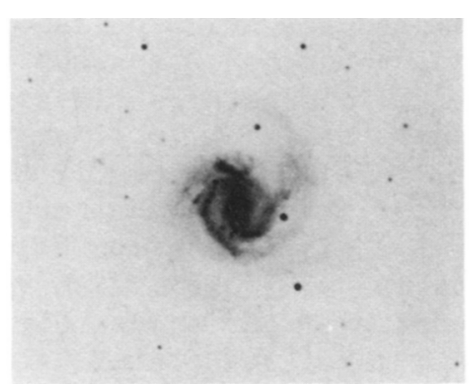

(b)

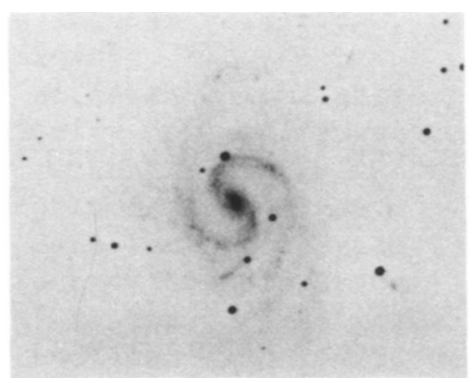

Figure 1. V-band images of (a) NGC 4303 and (b) NGC 4535 taken using the 105-cm Schmidt telescope at the Kiso Observatory. North is at the top.

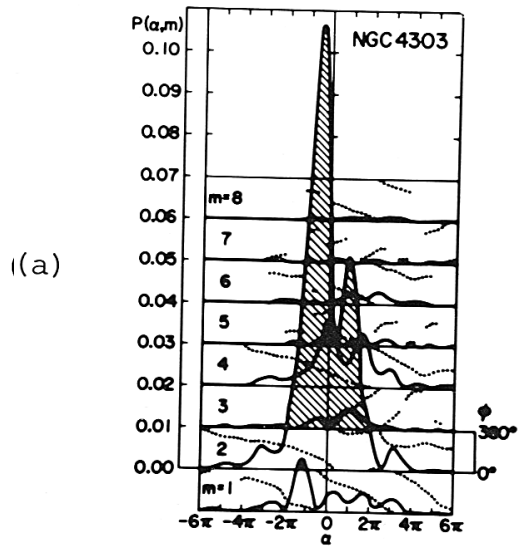

Figure 2. Normalized power spectra $P(\alpha, m)$ of (a) NGC 4303 and (b) NGC 4535. Spectra for $m=1,2, \ldots$, and 8 are shown by successively shifting 0.01 in the ordinate. Strong components with $P(\alpha, m) \geq 0.01$ are hatched. Dots are the phase angles $\phi$ of the corresponding $A(\alpha, m)$. The primary component of NGC 4303 is peaked at $P(-0.45 \pi, 2)$ and has a FWHM of $1.1 \pi$. The primary component of NGC 4535 is peaked at $P(0.25 \pi, 2)$ and has a FWHM of $0.9 \pi$.

(a)

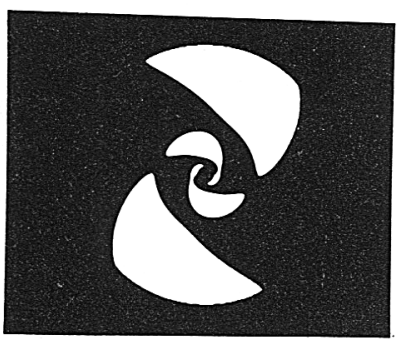

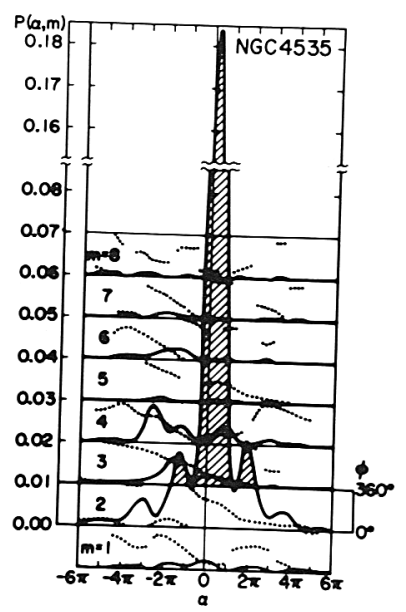

(b) (b)

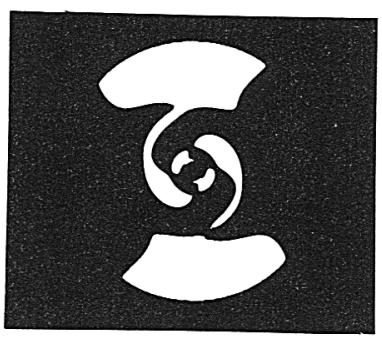

Figure 3. Patterns of the primary spectral components of (a) NGC 4303 and (b) NGC 4535. 